\title{
On potentially negative space time covariances obtained as sum of products of marginal ones *
}

\author{
P. Gregori†, E. Porcu $\dagger$, J. Mateu $\dagger \dagger$ Z. Sasvári† \\ † Department of Mathematics, Universitat Jaume I, Campus Riu Sec \\ E-12071 Castellón, Spain \\ ‡ Fachrichtung Mathematik, Institut für Mathematische Stochastik, Mommsenstr. 13 \\ 01602 Dresden, Germany
}

\begin{abstract}
Most of the literature on spatio-temporal covariance models proposes structures that are positive in the whole domain. However, problems of physical, biological or medical nature need covariance models allowing for negative values or oscillations from positive to negative values. In this paper we propose an easy-to-implement and interpretable class of models that admits this type of covariances. We show particular analytical examples that may be of interest in the biometrical context.

Keywords: Generalised product-sum model, Geostatistics, Nonseparability , Separability, Space-time covariance functions.
\end{abstract}

2000 Mathematics Subject Classification: 60G60, 62H11, 62M10, 62M40.

\section{Introduction}

The modellisation of biological or environmental phenomena which evolve in space and time has been one of the most important challenges over the last years. As pointed out by Janauer (2001) in an excellent paper, there is a strong interaction between biology and hydrology in the establishment, fluctuation and limitation of the aquatic environment in space and time. For instance, the analysis of the spatio-temporal distribution of flow is strongly related to the study of current velocities and turbulences. Particularly, some studies of the spatio-temporal correlation of turbulences have already been done for the

\footnotetext{
* Work partially funded by grants GV04A724 (Generalitat Valenciana) and MTM2004-06231 (Spanish Ministry of Science and Education)

${ }^{\dagger}$ Corresponding author. Email: mateu@mat.uji.es. Fax: +34.964.728429
} 
evaluation of kinematical and dynamical effects (Yakhot, Orszag and She, 1989). In another notable work in Levinson, Beall, Powers and Bengtson (1984), the problem of space time statistics of turbulences is faced up. In the merely temporal framework, it is worth citing $\mathrm{Xu}, \mathrm{Wu}, \mathrm{Huo}$ and $\mathrm{Wu}$ (2003) and $\mathrm{Xu}, \mathrm{Wu}$ and $\mathrm{Wu}$ (2003). On the other hand, in the spatial context, Shkarofsky (1968) emphasised the fact that in the study of turbulences it is often desirable to have covariance models allowing for negative values or oscillations from positive to negative values as the Euclidean distance tends to infinite. Unfortunately, most of previously proposed spatio-temporal covariance models in literature (see, among many others, Cressie and Huang, 1999; Gneiting, 2002; Christakos, 2000) are positive in the whole domain, so they are not useful for this purpose.

This strong motivation prompted our research. We are looking for space time covariance models satisfying two main features:

1. They may be negative or oscillate between negative and positive values.

2. They would preferable allow for an easy interpretation.

We believe it is reasonable to select a class of models satisfying property 2 , and then to answer, if possible, the natural question: can we obtain negative covariances starting from a class which is easy-to-implement and interpretable? The answer is yes and a satisfactory solution can be found through the theoretical results shown in this paper. The advantage is that, starting from a well-known class of models, one can implement, with a very simple procedure, covariance functions for space time data which can be negative in some part of their domain of definition. A direct consequence is that this kind of models can be used in many problems of physical, biological or environmental nature for which space time analysis is needed, such as in Pomeroy, Toth, Granger, Hedstrom and Essery (2003).

Summarising, this paper presents theoretical results answering the above mentioned questions, together with examples of potential interest on biomedical, environmental and hydrological problems. The plan of the paper is the following: Section 2 is dedicated to necessary background and notation about space time covariances and variograms. Section 3 is structured into three main parts regarding, respectively, our new proposal, some remarks on the Generalised Product-Sum model (De Cesare, Myers and Posa, 2000; 2001), and a new class of variograms for which a linear combination with negative parameters results in a permissible model. The paper ends with some conclusions and discussion.

\section{Theoretical background}

For the sake of conciseness, from now on we shall make reference to continuous weakly stationary Gaussian random fields $Z(\mathbf{x}, t), \mathbf{x} \in \mathbb{R}^{d}$ and $t \in \mathbb{R}$. Then, the function $\mathrm{C}_{s, t}(\mathbf{h}, u)=\operatorname{cov}\left(Z\left(\mathbf{x}_{i}, t_{i}\right), Z\left(\mathbf{x}_{j}, t_{j}\right)\right)$ is defined for $(\mathbf{h}, u)=\left(\mathbf{x}_{i}-\mathbf{x}_{j}, t_{i}-t_{j}\right) \in \mathbb{R}^{d} \times \mathbb{R}$ and is called the stationary spatio-temporal covariance function of the process, as it exclusively depends on the spatial and temporal separation vectors, respectively $\mathbf{h}$ and $u$. 
As well known, a real valued function $\mathrm{C}_{s, t}$ defined on the product space $\mathbb{R}^{d} \times \mathbb{R}$ is the covariance function associated to a stationary spatio-temporal random field if and only if

$$
\sum_{i=1}^{n} \sum_{j=1}^{n} a_{i} a_{j} \mathrm{C}_{s, t}\left(\mathbf{x}_{i}-\mathbf{x}_{j}, t_{i}-t_{j}\right) \geq 0
$$

for all finite sets of real coefficients $a_{i}$ and points $\left(\mathbf{x}_{i}, t_{i}\right) \in \mathbb{R}^{d} \times \mathbb{R}, i=1, \ldots, n$.

This property is called positive definiteness. With alternative notation, Christakos (2000, p.63) calls this property permissibility. A classical result in Bochner (1933) sets up the equivalence between covariance functions and characteristic functions of non-negative finite measures. Namely, a continuous function $\mathrm{C}_{s, t}$ as defined above is positive definite if and only if

$$
\mathrm{C}_{s, t}(\mathbf{h}, u)=\int_{\mathbb{R}^{d} \times \mathbb{R}} \mathrm{e}^{i\left(\boldsymbol{\omega}^{\prime} \mathbf{h}+\tau u\right)} \mathrm{d} F(\boldsymbol{\omega}, \tau),
$$

with $F$ a non-negative measure on $\mathbb{R}^{d} \times \mathbb{R}$.

A stationary covariance function is called isotropic if it is also rotation-invariant, i.e.

$$
\mathrm{C}_{s, t}\left(\mathbf{h}_{1}, u_{1}\right)=\mathrm{C}_{s, t}\left(\mathbf{h}_{2}, u_{2}\right) \quad \text { if } \quad\left\|\mathbf{h}_{1}\right\|=\left\|\mathbf{h}_{2}\right\| \quad \text { and } \quad\left|u_{1}\right|=\left|u_{2}\right|
$$

where $\|\cdot\|$ is the usual Euclidean norm on $\mathbb{R}^{d}$.

A space time covariance function is called separable if we can factor (Mitchell, Genton and Gumpertz, 2004)

$$
\mathrm{C}_{s, t}(\mathbf{h}, u)=\frac{\mathrm{C}_{s, t}(\mathbf{h}, 0) \mathrm{C}_{s, t}(\mathbf{0}, u)}{\mathrm{C}_{s, t}(\mathbf{0}, 0)} .
$$

In other words, separability means that the spatio-temporal covariance structure factors into a purely spatial and a purely temporal component, which allows for computationally efficient estimation and inference. Consequently, these models have been popular even in situations in which they may not be physically justifiable. An exhaustive simulation study of separable and nonseparable covariance models applied to medical problems can be found in Ibáñez-Gual and Simó (2004).

The variogram of a spatio-temporal intrinsically stationary random field is indicated with the symbol $\gamma_{s, t}$ and represents one half the variance of the increments of the process. Namely, we have

$$
\gamma_{s, t}(\mathbf{h}, u)=\frac{1}{2} \operatorname{Var}\left(Z\left(\mathbf{x}_{1}, t_{1}\right)-Z\left(\mathbf{x}_{2}, t_{2}\right)\right),
$$

where $(\mathbf{h}, u)=\left(\mathbf{x}_{1}-\mathbf{x}_{2}, t_{1}-t_{2}\right) \in \mathbb{R}^{d} \times \mathbb{R}$ has been previously defined as the spatio-temporal lag vector.

Recall that for well known results in Schoenberg (1938), variograms are conditionally negative definite functions, i.e.

$$
\sum_{i=1}^{n} \sum_{j=1}^{n} a_{i} a_{j} \gamma_{s, t}\left(\mathbf{x}_{i}-\mathbf{x}_{j}, t_{i}-t_{j}\right) \leq 0
$$


for any finite set of constants $a_{i}$ such that $\sum_{i=1}^{n} a_{i}=0$ and any finite set of points $\left(\mathbf{x}_{i}, t_{i}\right) \in \mathbb{R}^{d} \times \mathbb{R}, i=1,2, \ldots, n$.

The specialisation to the merely spatial or temporal case is straightforward. For the sake of clarity, from now on we shall indicate the merely spatial and temporal covariance and variogram respectively with the condensed notation $\mathrm{C}_{s}, \gamma_{s}, \mathrm{C}_{t}, \gamma_{t}$.

\section{Main results}

\subsection{The Generalised Sum-of-Products model and its negative covariances}

In this section we propose a tempting candidate class which can be obtained by considering the following setting: let $\left\{\mathrm{C}_{s i}: i=1,2, \ldots, n\right\}$ and $\left\{\mathrm{C}_{t i}: i=1,2, \ldots, n\right\}$ be, respectively, valid spatial and temporal stationary continuous and integrable covariance models, $n \in \mathbb{N}$. For $k_{1}, \ldots, k_{n} \geq 0$ let us define the function

$$
\mathrm{C}_{s, t}(\mathbf{h}, u)=\sum_{i=1}^{n} k_{i} \mathrm{C}_{s i}(\mathbf{h}) \mathrm{C}_{t i}(u), \quad(\mathbf{h}, u) \in \mathbb{R}^{d} \times \mathbb{R}
$$

This model defines a permissible stationary space time covariance in $\mathbb{R}^{d} \times \mathbb{R}$ having some desirable features, as it is positive definite for any positive setting of the parameters, it is integrable on $\mathbb{R}^{d} \times \mathbb{R}$ and admits a very easy implementation. Let us call this class with the acronym GSP, in order to denote Generalised Sum-of-Products. Once noticed that property 2 presented in the introduction is satisfied for this class, it is now natural to ask: is it possible to preserve positive definiteness even if some weights are set to be negative? In other words, we are trying to obtain covariances with negative values using the $G S P$ model with nonnegative covariances and negative weights. The answer can be found in the results subsequently shown.

Proposition 1. Let $\left\{\mathrm{C}_{s i}: i=1,2, \ldots, n\right\}$ and $\left\{\mathrm{C}_{t i}: i=1,2, \ldots, n\right\}$ be, respectively, valid spatial and temporal continuous and integrable covariance models, $n \in \mathbb{N}$. For $k_{1}, \ldots, k_{n} \in$ $\mathbb{R}$, consider the model in equation (1). Let us denote by $f_{s i}$ and $f_{t i}$, respectively and for $i=1,2, \ldots, n$, the Fourier transforms of covariances $\mathrm{C}_{s i}$ and $\mathrm{C}_{t i}$. Assume that we have at least one couple $\left(f_{s i}, f_{t i}\right)$ of non-vanishing functions, say $\left(f_{s n}, f_{t n}\right)$, and write

$$
m_{t i}:=\inf _{\tau \in \mathbb{R}} \frac{f_{t i}(\tau)}{f_{t n}(\tau)}, M_{t i}:=\sup _{\tau \in \mathbb{R}} \frac{f_{t i}(\tau)}{f_{t n}(\tau)}, m_{s i}:=\inf _{\boldsymbol{\omega} \in \mathbb{R}^{d}} \frac{f_{s i}(\boldsymbol{\omega})}{f_{s n}(\boldsymbol{\omega})}, M_{s i}:=\sup _{\boldsymbol{\omega} \in \mathbb{R}^{d}} \frac{f_{s i}(\boldsymbol{\omega})}{f_{s n}(\boldsymbol{\omega})} .
$$

Then we can state:

(i) If (1) is a permissible covariance then

$$
k_{n} \geq-\sum_{i=1}^{n-1} k_{i}\left[M_{s i} M_{t i} \mathbf{1}_{\left\{k_{i} \geq 0\right\}}+m_{s i} m_{t i} \mathbf{1}_{\left\{k_{i}<0\right\}}\right] .
$$


(ii) If $k_{n} \geq-\sum_{i=1}^{n-1} k_{i}\left[m_{s i} m_{t i} \mathbf{1}_{\left\{k_{i} \geq 0\right\}}+M_{s i} M_{t i} \mathbf{1}_{\left\{k_{i}<0\right\}}\right]$ then (1) is a permissible covariance.

(iii) For the special case $n=2,(1)$ is a permissible covariance if and only if

$$
k_{2} \geq-k_{1}\left[m_{s 1} m_{t 1} \mathbf{1}_{\left\{k_{1} \geq 0\right\}}+M_{s 1} M_{t 1} \mathbf{1}_{\left\{k_{1}<0\right\}}\right] .
$$

Proof. The Fourier transform of (1) can be expressed in terms of the respective Fourier transforms of the covariances

$$
f(\boldsymbol{\omega}, \tau)=\sum_{i=1}^{n} k_{i} f_{s i}(\boldsymbol{\omega}) f_{t i}(\tau)=f_{s n}(\boldsymbol{\omega}) f_{t n}(\tau)\left[\sum_{i=1}^{n-1} k_{i} \frac{f_{s i}(\boldsymbol{\omega})}{f_{s n}(\boldsymbol{\omega})} \frac{f_{t i}(\tau)}{f_{t n}(\tau)}+k_{n}\right]
$$

In order to prove (i), we have that $f(\boldsymbol{\omega}, \tau) \geq 0$ for all $\boldsymbol{\omega}$ and $\tau$. Then we can write

$$
0 \leq k_{n}+\sum_{i=1}^{n-1} k_{i} \frac{f_{s i}(\boldsymbol{\omega})}{f_{s n}(\boldsymbol{\omega})} \frac{f_{t i}(\tau)}{f_{t n}(\tau)} \leq k_{n}+\left[\sum_{\substack{i=1 \\ k_{i} \geq 0}}^{n-1} k_{i} M_{s i} M_{t i}+\sum_{\substack{i=1 \\ k_{i}<0}}^{n-1} k_{i} m_{s i} m_{t i}\right] .
$$

For (ii), using the assumption on $k_{n}$ we have

$$
\begin{aligned}
k_{n}+\sum_{i=1}^{n-1} k_{i} \frac{f_{s i}(\boldsymbol{\omega})}{f_{s n}(\boldsymbol{\omega})} \frac{f_{t i}(\tau)}{f_{t n}(\tau)} \geq & \sum_{\substack{i=1 \\
k_{i} \geq 0}}^{n-1} k_{i}\left(\frac{f_{s i}(\boldsymbol{\omega})}{f_{s n}(\boldsymbol{\omega})} \frac{f_{t i}(\tau)}{f_{t n}(\tau)}-m_{s i} m_{t i}\right) \\
& +\sum_{\substack{i=1 \\
k_{i}<0}}^{n-1} k_{i}\left(M_{s i} M_{t i}-\frac{f_{s i}(\boldsymbol{\omega})}{f_{s n}(\boldsymbol{\omega})} \frac{f_{t i}(\tau)}{f_{t n}(\tau)}\right) \geq 0 .
\end{aligned}
$$

Finally, in (iii) when $n=2$ we can sharpen the inequality used in (i) getting from $0 \leq f(\boldsymbol{\omega}, \tau)$, that $k_{2} \geq-k_{1} \frac{f_{s 1}(\boldsymbol{\omega})}{f_{s 2}(\boldsymbol{\omega})} \frac{f_{t_{1} 1}(\tau)}{f_{t 2}(\tau)}$ for all $\boldsymbol{\omega}$ and $\tau$, and hence

$$
k_{2} \geq-k_{1}\left[m_{s 1} m_{t 1} \mathbf{1}_{\left\{k_{1} \geq 0\right\}}+M_{s 1} M_{t 1} \mathbf{1}_{\left\{k_{1}<0\right\}}\right] .
$$

The converse is precisely (2).

Remark 1. A particular setting of the GSP class was considered by Ma (2005b), imposing the spatial covariances to belong to the same parametric family (and respectively for the temporal ones). Here we are working in a general setting, although obviously restricted to the feasibility of the computation of all those inf's and sup's, as we do not impose that the componentwise covariances belong to the same parametric family and only impose continuity and integrability, in order to ensure some stability properties involving the associated inverse Fourier transforms of the componentwise covariances. 
Remark 2. An interesting model that allows for attaining negative covariances was proposed by Vecchia (1988), under a very different setting than the one proposed in this paper. The author uses rational spectral densities whose associated covariance function results in a linear combination of modified Bessel functions of the second kind of order zero, that may attain negative values.

It should be stressed that his method only works on $\mathbb{R}^{2}$, whilst the method proposed in this paper and the one proposed by Ma (2005b) works on any d-dimensional Euclidean space and for spatial time series, allowing for a wider range of potential applications. Nevertheless, his approach is very interesting for the implications in estimation through likelihood methods.

Let us now show the simplest case $(n=2)$ of our previous result and discuss its applicability through some examples.

Corollary 1. Let $\mathrm{C}_{s i}$ and $\mathrm{C}_{t i}$ be valid spatial and temporal continuous and integrable covariance models, respectively, for $i=1,2$. For $\theta \in \mathbb{R}$ let us define the function

$$
\mathrm{C}_{s, t}(\mathbf{h}, u)=\theta \mathrm{C}_{s 1}(\mathbf{h}) \mathrm{C}_{t 1}(u)+(1-\theta) \mathrm{C}_{s 2}(\mathbf{h}) \mathrm{C}_{t 2}(u), \quad(\mathbf{h}, u) \in \mathbb{R}^{d} \times \mathbb{R}
$$

Let us denote by $f_{s i}$ and $f_{t i}$, respectively and for $i=1,2$, the Fourier transforms of covariances $\mathrm{C}_{t i}$ and $\mathrm{C}_{s i}$, assume the pair $\left(f_{s 2}, f_{t 2}\right)$ do not vanish, and write

$$
m_{t}:=\inf _{\tau \in \mathbb{R}} \frac{f_{t 1}(\tau)}{f_{t 2}(\tau)}, M_{t}:=\sup _{\tau \in \mathbb{R}} \frac{f_{t 1}(\tau)}{f_{t 2}(\tau)}, m_{s}:=\inf _{\boldsymbol{\omega} \in \mathbb{R}^{d}} \frac{f_{s 1}(\boldsymbol{\omega})}{f_{s 2}(\boldsymbol{\omega})}, M_{s}:=\sup _{\boldsymbol{\omega} \in \mathbb{R}^{d}} \frac{f_{s 1}(\boldsymbol{\omega})}{f_{s 2}(\boldsymbol{\omega})} .
$$

Then, $\mathrm{C}_{s, t}$ is a valid covariance if and only if

$$
\left[1-\max \left(1, M_{s} M_{t}\right)\right]^{-1} \leq \theta \leq\left[1-\min \left(1, m_{s} m_{t}\right)\right]^{-1}
$$

(where $0^{-1}=-\infty$ and $(-\infty)^{-1}=0$ in the left hand side, and $0^{-1}=+\infty$ in the right hand side).

For the proof, write $k_{1}=\theta, k_{2}=1-\theta$ and use the description of equation (2).

It is worth mentioning the specialised result for merely spatial covariances as shown in the following corollary.

Corollary 2. Let $\mathrm{C}_{s i}$ be valid spatial continuous and integrable covariance models for $i=1,2$. For $\theta \in \mathbb{R}$ let us define the function

$$
\mathrm{C}_{s}(\mathbf{h})=\theta \mathrm{C}_{s 1}(\mathbf{h})+(1-\theta) \mathrm{C}_{s 2}(\mathbf{h}), \quad \mathbf{h} \in \mathbb{R}^{d} .
$$

Let us denote by $f_{s i}$ the Fourier transforms of covariances $\mathrm{C}_{t i}$, assume $f_{s 2}$ does not vanish and write

$$
m:=\inf _{\boldsymbol{\omega} \in \mathbb{R}^{d}} \frac{f_{s 1}(\boldsymbol{\omega})}{f_{s 2}(\boldsymbol{\omega})}, \quad M:=\sup _{\boldsymbol{\omega} \in \mathbb{R}^{d}} \frac{f_{s 1}(\boldsymbol{\omega})}{f_{s 2}(\boldsymbol{\omega})} .
$$


Then, $\mathrm{C}_{s}$ is a valid spatial covariance if and only if

$$
[1-\max (1, M)]^{-1} \leq \theta \leq[1-\min (1, m)]^{-1}
$$

(where $0^{-1}=-\infty$ and $(-\infty)^{-1}=0$ in the left hand side, and $0^{-1}=+\infty$ in the right hand side).

Example 1. In order to show the potentiality of the above presented results, in particular of those in Corollaries 1 and 2, we restrict ourselves to the case of Gaussian and Matérn (Matérn, 1986) families, for which we have analytical expressions for the Fourier transforms.

We write $\mathbf{x} \in \mathbb{R}^{k}$ indistinctly for space and time component (i.e., $\mathbf{x} \equiv \mathbf{h} \in \mathbb{R}^{d}$ or $\mathbf{x} \equiv u \in \mathbb{R})$. If

$$
\begin{aligned}
& \mathrm{C}_{G}(\mathbf{x})=\mathrm{C}_{G}\left(\mathbf{x} \mid \sigma_{G}, \alpha_{G}\right)=\sigma_{G}^{2} \exp \left(-\alpha_{G}\|\mathbf{x}\|^{2}\right) \\
& \mathrm{C}_{M}(\mathbf{x})=\mathrm{C}_{M}\left(\mathbf{x} \mid \sigma_{M}, \nu, \alpha_{M}\right)=\sigma_{M}^{2}\left(\alpha_{M}\|x\|\right)^{\nu} \mathcal{K}_{\nu}\left(\alpha_{M}\|x\|\right)
\end{aligned}
$$

are, respectively, the Gauss and Matérn covariance functions $\left(\mathcal{K}_{\nu}\right.$ is the modified Bessel function of the second kind of order $\nu$ ), then

$$
\begin{aligned}
& f_{G}(\boldsymbol{\omega})=\sigma_{G}^{2} \pi^{d / 2} \alpha_{G}^{-d / 2} \exp \left(-\frac{1}{4 \alpha_{G}}\|\boldsymbol{\omega}\|^{2}\right) \\
& f_{M}(\boldsymbol{\omega})=\sigma_{M}^{2} 2^{\nu-1} \pi^{-d / 2} \Gamma\left(\nu+\frac{d}{2}\right) \alpha_{M}^{2 \nu}\left(\alpha_{M}^{2}+\|\boldsymbol{\omega}\|^{2}\right)^{-\nu-\frac{d}{2}}
\end{aligned}
$$

for $\boldsymbol{\omega} \in \mathbb{R}^{k}$, are their respective Fourier transforms (see, for instance, Yaglom, 1986, p.363). We show in Table 1 the results of $m$ and $M$ (subscripted in each case with the initials of the pair of used covariances) for the four possible quotients of Fourier transforms, setting up the following notation for the cases:

Firstly, let $\mathrm{C}_{G_{i}}(\mathbf{x})=\mathrm{C}_{G_{i}}\left(\mathbf{x} \mid \sigma_{i}, \alpha_{i}\right)=\sigma_{i}^{2} \exp \left(-\alpha_{i}\|\mathbf{x}\|^{2}\right)$ for $i=1,2$ for the Gauss vs Gauss case. Secondly, let $\mathrm{C}_{G}(\mathbf{x})=\mathrm{C}_{G}\left(\mathbf{x} \mid \sigma_{G}, \alpha_{G}\right)=\sigma_{G}^{2} \exp \left(-\alpha_{G}\|\mathbf{x}\|^{2}\right)$ and $\mathrm{C}_{M}(\mathbf{x})=$ $\mathrm{C}_{M}\left(\mathbf{x} \mid \sigma_{M}, \nu, \alpha_{M}\right)=\sigma_{M}^{2}\left(\alpha_{M}\|x\|\right)^{\nu} \mathcal{K}_{\nu}\left(\alpha_{M}\|x\|\right)$ for the Gauss vs Matérn case, and thirdly, let $\mathrm{C}_{M_{i}}(\mathbf{x})=\mathrm{C}_{M_{i}}\left(\mathbf{x} \mid \sigma_{i}, \nu_{i}, \alpha_{i}\right)=\sigma_{i}^{2}\left(\alpha_{i}\|x\|\right)^{\nu_{i}} \mathcal{K}_{\nu_{i}}\left(\alpha_{i}\|x\|\right)$ for the Matérn vs Matérn case.

Computations are direct since the quotient of Fourier transforms is a one variable function depending on a positive real argument $z=\|\boldsymbol{\omega}\|^{2}$. For instance, for the Matérn vs Matérn case, the quotient is

$$
\left(\frac{\sigma_{1}}{\sigma_{2}}\right)^{2} 2^{\nu_{1}-\nu_{2}} \frac{\Gamma\left(\nu_{1}+\frac{d}{2}\right)}{\Gamma\left(\nu_{2}+\frac{d}{2}\right)} \frac{\alpha_{1}^{2 \nu}}{\alpha_{2}^{2 \nu}} \frac{\left(\alpha_{1}^{2}+z\right)^{-\nu_{1}-\frac{d}{2}}}{\left(\alpha_{2}^{2}+z\right)^{-\nu_{2}-\frac{d}{2}}}
$$

Then optimization is easily reached by the evaluation of that quotient at $z=0, z=\infty$ and at the critical points found (by solving where the derivative vanishes) in each case.

Finally, observe that $m_{M, G}$ and $M_{M, G}$ (for the Matérn vs Gauss case) can be computed as $m_{M, G}=M_{G, M}^{-1}$ and $M_{M, G}=m_{G, M}^{-1}$ (see Table 1$)$. 
Table 1: Results of inf's and sup's needed in Proposition 1 and Corollaries 1 and 2 for the particular cases of using Gaussian vs. Gaussian, Gaussian vs. Matérn and Matérn vs. Matérn covariance functions, respectively, in terms of their parameters values. Here $k$ denotes the temporal or spatial dimension (respectively 1 or $d$ ).

\begin{tabular}{|c|c|c|}
\hline \multicolumn{3}{|c|}{ Gauss $_{1} /$ Gauss $_{2}$} \\
\hline Parameters & $m_{G_{1}, G_{2}}$ & $M_{G_{1}, G_{2}}$ \\
\hline $0<\alpha_{1}<\alpha_{2}$ & 0 & $\left(\frac{\sigma_{1}}{\sigma_{2}}\right)^{2}\left(\frac{\alpha_{2}}{\alpha_{1}}\right)^{k / 2}$ \\
\hline$\alpha_{1} \geq \alpha_{2}>0$ & $\left(\frac{\sigma_{1}}{\sigma_{2}}\right)^{2}\left(\frac{\alpha_{2}}{\alpha_{1}}\right)^{k / 2}$ & $+\infty$ \\
\hline \multicolumn{3}{|c|}{ Gauss/Matérn } \\
\hline Parameters & $m_{G, M}$ & $M_{G, M}$ \\
\hline$\frac{\alpha_{M}^{2}}{\left(\nu+\frac{k}{2}\right)}>4 \alpha_{G}$ & 0 & $\begin{array}{l}\left(\frac{\sigma_{G}}{\sigma_{M}}\right)^{2}\left(\frac{\pi \alpha_{M}^{2}}{\alpha_{G}}\right)^{k / 2} 2^{-\nu+1} \\
\quad \times \Gamma\left(\nu+\frac{k}{2}\right)^{-1}\end{array}$ \\
\hline$\frac{\alpha_{M}^{2}}{\left(\nu+\frac{k}{2}\right)} \leq 4 \alpha_{G}$ & 0 & $\begin{array}{l}\left(\frac{\sigma_{G}}{\sigma_{M}}\right)^{2} 2^{k+1} \pi^{k / 2}\left(\frac{2 \alpha_{G}}{\alpha_{M}^{2}}\right)^{\nu} \\
\quad \times \frac{\left(\nu+\frac{k}{2}\right)^{\nu+\frac{k}{2}}}{\Gamma\left(\nu+\frac{k}{2}\right)} \\
\quad \times \exp \left(\frac{\alpha_{M}^{2}}{4 \alpha_{G}}-\left(\nu+\frac{k}{2}\right)\right) \\
\end{array}$ \\
\hline \multicolumn{3}{|c|}{ Matérn $_{1} /$ Matérn $_{2}$} \\
\hline Parameters & $m_{M_{1}, M_{2}}$ & $M_{M_{1}, M_{2}}$ \\
\hline $\begin{array}{c}\nu_{1}<\nu_{2} \\
\frac{\alpha_{2}^{2}}{\alpha_{1}^{2}} \leq \frac{\nu_{2}+\frac{k}{2}}{\nu_{1}+\frac{k}{2}}\end{array}$ & $\left(\frac{\sigma_{G}}{\sigma_{M}}\right)^{2} \frac{\Gamma\left(\nu_{1}+\frac{k}{2}\right)}{\Gamma\left(\nu_{2}+\frac{k}{2}\right)} 2^{\nu_{1}-\nu_{2}}\left(\frac{\alpha_{2}}{\alpha_{1}}\right)^{k}$ & $+\infty$ \\
\hline $\begin{aligned} & \nu_{1}<\nu_{2} \\
& \frac{\alpha_{2}^{2}}{\alpha_{1}^{2}}>\frac{\nu_{2}+\frac{k}{2}}{\nu_{1}+\frac{k}{2}}\end{aligned}$ & $\begin{array}{l}\left(\frac{\sigma_{G}}{\sigma_{M}}\right)^{2} \frac{\Gamma\left(\nu_{1}+\frac{k}{2}\right)}{\Gamma\left(\nu_{2}+\frac{k}{2}\right)} \frac{\left(\nu_{1}+\frac{k}{2}\right)^{\nu_{2}+\frac{k}{2}}}{\left(\nu_{2}+\frac{k}{2}\right)^{\nu_{1}+\frac{k}{2}}} \\
\quad \times\left(\frac{\alpha_{2}^{2}-\alpha_{1}^{2}}{2\left(\nu_{2}-\nu_{1}\right)}\right)^{\nu_{2}-\nu_{1}} \frac{\alpha_{1}^{2 \nu_{1}}}{\alpha_{2}^{2 \nu_{2}}}\end{array}$ & $+\infty$ \\
\hline$\nu_{1}=\nu_{2}$ and $\alpha_{2} \geq \alpha_{1}$ & $\left(\frac{\sigma_{G}}{\sigma_{M}}\right)^{2}\left(\frac{\alpha_{1}}{\alpha_{2}}\right)^{2 \nu_{1}}$ & $\left(\frac{\sigma_{G}}{\sigma_{M}}\right)^{2}\left(\frac{\alpha_{2}}{\alpha_{1}}\right)^{k}$ \\
\hline$\nu_{1}=\nu_{2}$ and $\alpha_{2}<\alpha_{1}$ & $\left(\frac{\sigma_{G}}{\sigma_{M}}\right)^{2}\left(\frac{\alpha_{2}}{\alpha_{1}}\right)^{k}$ & $\left(\frac{\sigma_{G}}{\sigma_{M}}\right)^{2}\left(\frac{\alpha_{1}}{\alpha_{2}}\right)^{2 \nu_{1}}$ \\
\hline $\begin{array}{c}\nu_{1}>\nu_{2} \\
\frac{\alpha_{2}^{2}}{\alpha_{1}^{2}}<\frac{\nu_{2}+\frac{k}{2}}{\nu_{1}+\frac{k}{2}}\end{array}$ & 0 & $\begin{array}{c}\left(\frac{\sigma_{G}}{\sigma_{M}}\right)^{2} \frac{\Gamma\left(\nu_{1}+\frac{k}{2}\right)}{\Gamma\left(\nu_{2}+\frac{k}{2}\right)} \frac{\left(\nu_{1}+\frac{k}{2}\right)^{\nu_{2}+\frac{k}{2}}}{\left(\nu_{2}+\frac{k}{2}\right)^{\nu_{1}+\frac{k}{2}}} \\
\quad \times\left(\frac{\alpha_{2}^{2}-\alpha_{1}^{2}}{2\left(\nu_{2}-\nu_{1}\right)}\right)^{\nu_{2}-\nu_{1}} \frac{\alpha_{1}^{2 \nu_{1}}}{\alpha_{2}^{2 \nu_{2}}}\end{array}$ \\
\hline $\begin{array}{c}\nu_{1}>\nu_{2} \\
\frac{\alpha_{2}^{2}}{\alpha_{1}^{2}} \geq \frac{\nu_{2}+\frac{k}{2}}{\nu_{1}+\frac{k}{2}}\end{array}$ & 0 & $\left(\frac{\sigma_{G}}{\sigma_{M}}\right)^{2} \frac{\Gamma\left(\nu_{1}+\frac{k}{2}\right)}{\Gamma\left(\nu_{2}+\frac{k}{2}\right)} 2^{\nu_{1}-\nu_{2}}\left(\frac{\alpha_{2}}{\alpha_{1}}\right)^{k}$ \\
\hline
\end{tabular}


Now we are in position to consider linear combinations of marginal covariances within these families taking the form

$$
\mathrm{C}_{s, t}(\mathbf{h}, u)=\theta \mathrm{C}_{s 1}(\mathbf{h}) \mathrm{C}_{t 1}(u)+(1-\theta) \mathrm{C}_{s 2}(\mathbf{h}) \mathrm{C}_{t 2}(u), \quad(\mathbf{h}, u) \in \mathbb{R}^{d} \times \mathbb{R}
$$

and characterise the exact interval of $\theta$ for which $\mathrm{C}_{s, t}$ is a valid covariance, in terms of the parameter values of the particular covariances taking part in the expression. For instance, if we take

$$
\begin{array}{ll}
\mathrm{C}_{s 1}(\mathbf{h})=\sigma_{s 1}^{2} \exp \left(-\alpha_{1}\|\mathbf{h}\|^{2}\right) & \mathrm{C}_{s 2}(\mathbf{h})=\sigma_{s 2}^{2} \exp \left(-\alpha_{2}\|\mathbf{h}\|\right) \\
\mathrm{C}_{t 1}(u)=\sigma_{t 1}^{2}\left(\beta_{1}|u|\right)^{\nu} \mathcal{K}_{\nu}\left(\beta_{1}|u|\right) & \mathrm{C}_{t 2}(u)=\sigma_{t 2}^{2} \exp \left(-\beta_{2}|u|\right)
\end{array}
$$

with space dimension $d=2, \alpha_{2}^{2}>6 \alpha_{1}, \nu=1$ and $\beta_{1}^{2}>\frac{3}{2} \beta_{2}^{2}$ then, we can apply Corollary 1 , and the results of Table 1, in order to state that the linear combination previously written is a valid covariance if and only if

$$
\left[1-\max \left(1, \frac{2 \pi \sigma_{s 1}^{2} \sigma_{t 1}^{2} \alpha_{2}^{2} \beta_{2}}{\sigma_{s 2}^{2} \sigma_{t 2}^{2} \alpha_{1} \beta_{1}}\right)\right]^{-1} \leq \theta \leq 1
$$

(resulting in the interval $\theta \leq 1$ whenever the last maximum equals to 1 ).

Using these tools, any other combination of covariances can be analysed by the interested reader. It is worth emphasising that the example applies to the covariances associated to the Ornstein-Uhlembeck process (i.e. the exponential family) and to the so-called spherical model, since they are both particular cases of the Matérn family, respectively for $\nu=1 / 2$ and $\nu=3 / 2$. Figure 1 illustrates the features of this type of construction in the one dimensional case.

Remark 3. Theorems 1 and 2 in Ma (2005a) as well as Theorem 3 and Corollary 3.1 in Ma (2005b), can be obtained by using computation technique in Example 1.

\subsection{The Generalised Product-Sum model: a cautionary example}

A well-known class of covariance models having a notably easy physical interpretation was proposed by De Cesare, Myers and Posa (2000), later reconsidered by De Iaco, Myers and Posa (2001) and called Generalised Product-Sum (GPS) model. The authors showed how to obtain spatio-temporal covariances through weighted sums and products of marginal covariance functions, where the weights must be nonnegative in order to preserve the permissibility of the resulting covariance function. The GPS model has inspired a growing literature based on what is called mixture-based covariances. Among other authors, it is worth citing Ma (2002) with mixtures of stationary covariance functions and Porcu, Saura and Mateu (2005) with the so called Mixed Forms. The GPS class of valid product-sum covariance models admits, in its general formulation, the following equation

$$
\mathrm{C}_{s, t}(\mathbf{h}, u)=k_{1} \mathrm{C}_{s}(\mathbf{h}) \mathrm{C}_{t}(u)+k_{2} \mathrm{C}_{s}(\mathbf{h})+k_{3} \mathrm{C}_{t}(u)
$$



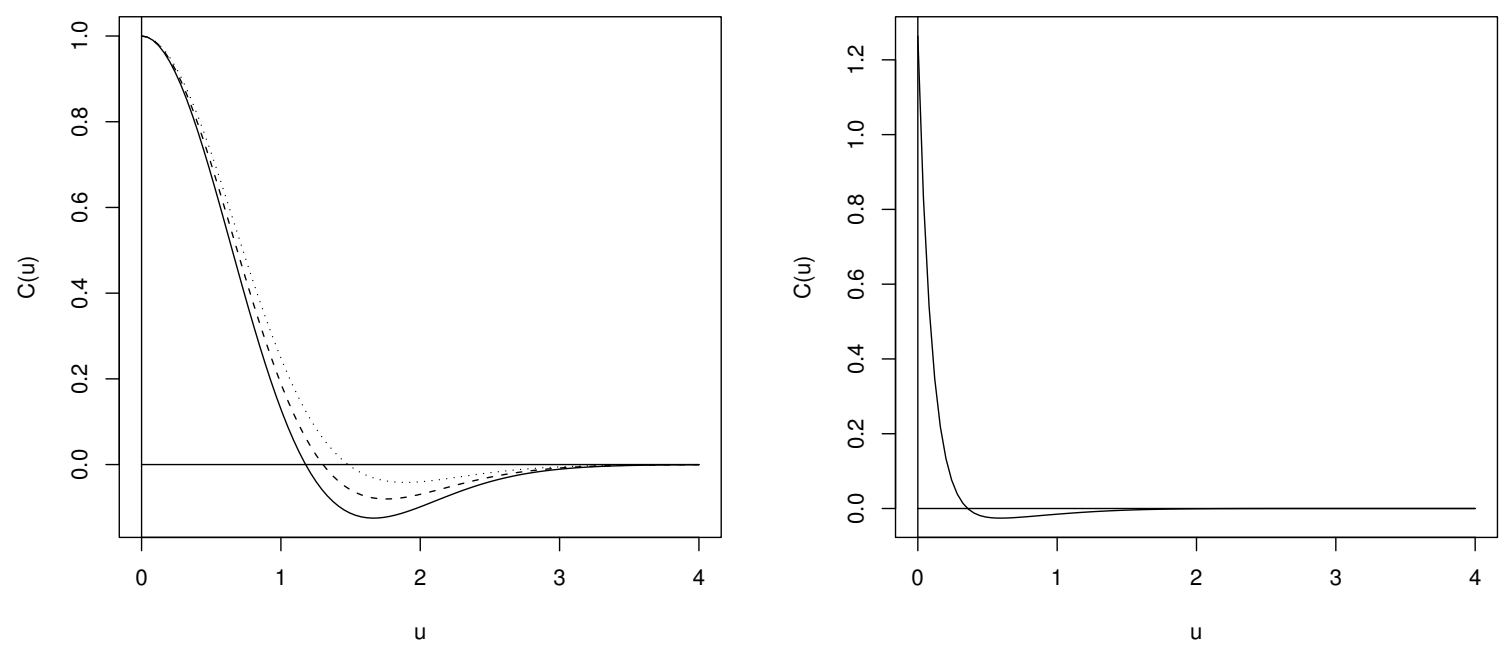

Figure 1: Left: Temporal covariance resulting from the linear combination of two Gaussian covariances $\left(\alpha_{1}=0.5, \alpha_{2}=1\right)$, with $\theta=-1$ (solid), -0.75 (dashed), -0.5 (dotted). Right: Temporal covariance resulting from the linear combination of a Gaussian and a Matérn covariances $\left(\alpha_{G}=1, \nu=0.5, \alpha_{M}=\sqrt{100}\right)$, with $\theta=-0.04$.

where $\mathrm{C}_{t}$ and $\mathrm{C}_{s}$ are valid temporal and spatial covariance models, respectively. For positive definiteness, it is then sufficient that $k_{1}>0, k_{2} \geq 0$ and $k_{3} \geq 0$.

As previously said, this model has been appreciated in literature for its flexibility, ease of construction and physical interpretation. Starting from (3), it is easy to see, using well-known relationships between the covariance and variogram, that the corresponding $G P S$ spatio-temporal variogram would take the form

$$
\gamma_{s, t}(\mathbf{h}, u)=\left(k_{2}+k_{1} \mathrm{C}_{t}(0)\right) \gamma_{s}(\mathbf{h})+\left(k_{3}+k_{1} \mathrm{C}_{s}(\mathbf{0})\right) \gamma_{t}(u)-k_{1} \gamma_{s}(\mathbf{h}) \gamma_{t}(u),
$$

provided that $\gamma_{s}$ and $\gamma_{t}$ are bounded. The importance of the GPS model is that it opened a wide literature regarding space time covariances obtained through mixture modelling. De Iaco, Myers and Posa (2001), with some algebraic manipulation, showed that model (4) can be rewritten as

$$
\gamma_{s, t}(\mathbf{h}, u)=\gamma_{s, t}(\mathbf{h}, 0)+\gamma_{s, t}(\mathbf{0}, u)-k \gamma_{s, t}(\mathbf{h}, 0) \gamma_{s, t}(\mathbf{0}, u)
$$

The authors proved that a necessary and sufficient condition for positive definiteness of this model is that $0 \leq k \leq 1 / \max \left(\operatorname{sill}\left(\gamma_{s, t}(\mathbf{h}, 0)\right)\right.$; $\left.\operatorname{sill}\left(\gamma_{s, t}(\mathbf{0}, u)\right)\right)$, where sill $(\cdot)$ stands for the variance of the random process associated to the marginal variogram in its argument.

Unfortunately, due to its lack of integrability on $\mathbb{R}^{d} \times \mathbb{R}$, this model cannot be treated in this paper, even if possessing some attractive features. The reasons for which we shall not make use of this model will be clarified in the sequel. But firstly we aim to remark that this model is not strictly positive in some pathological situations for which we invite to caution. For the sake of clarity, we shall make reference to the merely $\mathbb{R}^{2}$ spatial setting 
and follow the beautiful idea of Myers and Journel (1990). Suppose to have only four sampling points in a compact domain of $\mathbb{R}^{2}$, say $\left.\{(0,0),(x, 0),(0, y),(x, y))\right\}$, with $x, y$ positive scalars. Suppose we are using a model of the GPS class having equation

$$
\gamma(x, y)=\gamma_{1 s}(x, 0)+\gamma_{2 s}(0, y)-k \gamma_{1 s}(x, 0) \gamma_{2 s}(0, y)
$$

where $\operatorname{sill}\left(\gamma_{1 s}(x, 0)\right)=\operatorname{sill}\left(\gamma_{2 s}(0, y)\right)=1$ and $\gamma_{1 s}, \gamma_{2 s}$ not strictly increasing (but permissible) variograms respectively on $x$ and $y$ (it is not compulsory for the variogram to be an increasing function of the lag vector as can be seen in Chilès and Delfiner, 1999, Sect. 2.5). Finally, setting $k=1$ and supposing that $\gamma_{1 s}(x, 0)=a, \gamma_{2 s}(0, y)=b$, with $a=b /(b-1)$ and $b>1$, it is easy to see that the resulting variogram matrix would take the form

$$
\left(\begin{array}{lllll}
0 & a & b & 0 & 1 \\
a & 0 & 0 & b & 1 \\
b & 0 & 0 & a & 1 \\
0 & b & a & 0 & 1 \\
1 & 1 & 1 & 1 & 0
\end{array}\right) .
$$

Now, it can be seen that the determinant of this matrix is zero, thus the variogram and covariance matrices are singular, with all the consequences related to the kriging interpolation procedures which strictly depend on this matrices and on their inverses.

The extension to the spatio-temporal case is straightforward and various counterexamples of this type can be proposed working in a similar setting. Concluding, we recommend using this model only with parametric variograms which are strictly increasing with respect to the Euclidean spatial and temporal distance.

The GPS model in equation (3) is not integrable in $\mathbb{R}^{d} \times \mathbb{R}$. It implies that the technique of Fourier transform can not be used to find out the complete domain of parameters $\left(k_{1}, k_{2}, k_{3}\right)$ giving valid covariances. However, by means of two auxiliary continuous integrable covariances $\mathrm{C}_{s 2}, \mathrm{C}_{t 2}$ we can build the model

$$
\widetilde{\mathrm{C}}_{s, t}(\mathbf{h}, u)=k_{1} \mathrm{C}_{s}(\mathbf{h}) \mathrm{C}_{t}(u)+k_{2} \mathrm{C}_{s}(\mathbf{h}) \mathrm{C}_{t 2}(u)+k_{3} \mathrm{C}_{t}(u) \mathrm{C}_{s 2}(\mathbf{h}), \quad(\mathbf{h}, u) \in \mathbb{R}^{d} \times \mathbb{R}
$$

which is integrable, and use the technique of Corollary 1 to show that the domain of valid parameters is

$$
\begin{aligned}
D= & \left\{\left(\kappa_{1}, \kappa_{2}, \kappa_{3}\right): \kappa_{2} \geq 0, \kappa_{3} \geq 0, \kappa_{1} \geq-m_{t} \kappa_{2}-m_{s} \kappa_{3}\right\} \\
& \cup\left\{\left(\kappa_{1}, \kappa_{2}, \kappa_{3}\right): \kappa_{2} \geq 0, \kappa_{3}<0, \kappa_{1} \geq-m_{t} \kappa_{2}-M_{s} \kappa_{3}\right\} \\
& \cup\left\{\left(\kappa_{1}, \kappa_{2}, \kappa_{3}\right): \kappa_{2}<0, \kappa_{3} \geq 0, \kappa_{1} \geq-M_{t} \kappa_{2}-m_{s} \kappa_{3}\right\} \\
& \cup\left\{\left(\kappa_{1}, \kappa_{2}, \kappa_{3}\right): \kappa_{2}<0, \kappa_{3}<0, \kappa_{1} \geq-M_{t} \kappa_{2}-M_{s} \kappa_{3}\right\}
\end{aligned}
$$

where

$$
m_{t}:=\inf _{\tau \in \mathbb{R}} \frac{f_{t 2}(\tau)}{f_{t}(\tau)}, M_{t}:=\sup _{\tau \in \mathbb{R}} \frac{f_{t 2}(\tau)}{f_{t}(\tau)}, m_{s}:=\inf _{\boldsymbol{\omega} \in \mathbb{R}^{d}} \frac{f_{s 2}(\boldsymbol{\omega})}{f_{s}(\boldsymbol{\omega})}, M_{s}:=\sup _{\boldsymbol{\omega} \in \mathbb{R}^{d}} \frac{f_{s 2}(\boldsymbol{\omega})}{f_{s}(\boldsymbol{\omega})} .
$$


If we use sequences $\mathrm{C}_{s 2}^{(n)}, \mathrm{C}_{t 2}^{(n)}$ of covariances converging pointwise to constant one, we would have a sequence $\widetilde{\mathrm{C}}_{s t}^{(n)}$ of continuous integrable covariances belonging to the GSP class and converging pointwise to the generalised product-sum model. For each $n$ we could compute exactly the domain $D^{(n)}$ of validity of $\mathrm{C}_{s t}^{(n)}$. But as $n \rightarrow \infty$,

$$
m_{s}^{(n)} \rightarrow 0, \quad M_{s}^{(n)} \rightarrow \infty, \quad m_{t}^{(n)} \rightarrow 0, \quad M_{t}^{(n)} \rightarrow \infty
$$

and then we see that the domain $D^{(n)}$ tends to be the first octant $\mathbb{R}_{+}^{3}$ as $n \rightarrow \infty$.

\subsection{Variograms through particular linear combinations of Bern- stein functions with negative weights}

Until now we have been working with the model (1) which is expressed in terms of covariances. The research for linear combinations with negative weights can also be addressed to variograms. Before considering this framework, let us give some necessary background about completely monotone and Bernstein functions. A completely monotone function $\varphi$ is a positive decreasing function defined on the positive real line satisfying

$$
(-1)^{n} \varphi^{(n)}(t)>0, \quad t>0
$$

for all positive natural number $n$. Much more can be said about this class of functions, but it is beyond the scope of this paper. For a complete review on completely monotone functions, we refer the reader to the excellent book of Berg and Forst (1975).

Bernstein functions $\psi$ are the integrals or derivatives of completely monotone functions. By well-known results, this class of functions admits the integral representation

$$
\psi(t)=\int_{0}^{\infty}\left(1-e^{-t u}\right) u^{-1} \mathrm{~d} F(u),
$$

provided that $\int_{0}^{1} u^{-1} \mathrm{~d} F(u)$ exists and is finite. By Schoenberg's (1938) Theorem, an isotropic variogram is permissible in any $d$-dimensional space $\mathbb{R}^{d}$ if and only if it is of the form

$$
\gamma_{s}(\mathbf{h})=\psi\left(\|\mathbf{h}\|^{2}\right) .
$$

We are particularly interested in the consideration of the following two classes of zonally and geometrically anisotropic variograms

$$
\gamma_{s, t}(\mathbf{h}, u)=\sum_{i=1}^{n} k_{i} \psi\left(\alpha_{i}\|\mathbf{h}\|^{2}+\beta_{i}|u|^{2}\right)
$$

and

$$
\gamma_{s, t}(\mathbf{h}, u)=\sum_{i=1}^{n} k_{i} \psi\left(\alpha_{i}\|\mathbf{h}\|+\beta_{i}|u|\right)
$$


These classes are particularly important as they join the celebrated geometrical anisotropic approach in Dimitrakopoulos and Luo (1994) with another well-known approach for zonal anisotropy, the nested model, as in Rohuani and Hall (1989). Starting from these classes, we show in the next proposition how to obtain valid variograms through linear combinations that can have negative weights, extending Ma (2005a, Thm. 3) to the spatio-temporal case.

Proposition 2. Let $\left\{\alpha_{i}\right\}_{i=1}^{n}$ and $\left\{\beta_{i}\right\}_{i=1}^{n}$ be two increasing finite sequences of positive constants and $\psi$ a Bernstein function. For $k_{1}, \ldots, k_{n} \in \mathbb{R}$, we have that:

1. The model in equation (6) is a permissible intrinsically stationary variogram defined on $\mathbb{R}^{d} \times \mathbb{R}$ if

$$
k_{n} \geq-\sum_{i=1}^{n-1}\left(\frac{\alpha_{n}}{\alpha_{i}}\right)^{d / 2}\left(\frac{\beta_{n}}{\beta_{i}}\right)^{1 / 2} \mathbf{1}_{\left\{k_{i}<0\right\}}
$$

2. The model in equation (7) is a permissible intrinsically stationary variogram defined on $\mathbb{R}^{d} \times \mathbb{R}$ if

$$
k_{n} \geq-\sum_{i=1}^{n-1} \frac{\alpha_{i} \beta_{i}}{\alpha_{n} \beta_{n}} \mathbf{1}_{\left\{k_{i} \geq 0\right\}}+\left(\frac{\alpha_{n}}{\alpha_{i}}\right)^{d}\left(\frac{\beta_{n}}{\beta_{i}}\right) \mathbf{1}_{\left\{k_{i}<0\right\}}
$$

Proof. For 1$)$ it is sufficient to show that $\sum_{i=1}^{m} \sum_{j=1}^{m} a_{i} a_{j} \gamma\left(\mathbf{x}_{i}-\mathbf{x}_{j}, t_{i}-t_{j}\right) \leq 0$ for arbitrary families $\left\{\left(\mathbf{x}_{i}, t_{i}\right)\right\}_{i=1}^{m} \subset \mathbb{R}^{d} \times \mathbb{R}$ and $\left\{a_{i}\right\}_{i=1}^{n} \subset \mathbb{R}$ under the restriction $\sum_{i=1}^{m} a_{i}=0$. Thus using equation (5) we get

$$
\begin{aligned}
& \sum_{i=1}^{m} \sum_{j=1}^{m} a_{i} a_{j} \gamma\left(\mathbf{x}_{i}-\mathbf{x}_{j}, t_{i}-t_{j}\right) \\
& =\sum_{i=1}^{m} \sum_{j=1}^{m} a_{i} a_{j} \sum_{l=1}^{n} k_{l} \int_{0}^{\infty}\left(1-\exp \left(-\alpha_{l}\left\|\mathbf{x}_{i}-\mathbf{x}_{j}\right\|^{2} v-\beta_{l}\left|t_{i}-t_{j}\right|^{2} v\right)\right) v^{-1} \mathrm{~d} F(v) \\
& =\sum_{l=1}^{n} k_{l} \int_{0}^{\infty} \sum_{i=1}^{m} \sum_{j=1}^{m} a_{i} a_{j}\left(1-\exp \left(-\alpha_{l}\left\|\mathbf{x}_{i}-\mathbf{x}_{j}\right\|^{2} v-\beta_{l}\left|t_{i}-t_{j}\right|^{2} v\right)\right) v^{-1} \mathrm{~d} F(v) \\
& =-\sum_{i=1}^{m} \sum_{j=1}^{m} a_{i} a_{j} \int_{0}^{\infty} \sum_{l=1}^{n} k_{l} \mathrm{C}_{s, t}^{v, l}\left(\mathbf{x}_{i}-\mathbf{x}_{j}, t_{i}-t_{j}\right) v^{-1} \mathrm{~d} F(v)
\end{aligned}
$$

where $\mathrm{C}_{s, t}^{v, l}(\mathbf{h}, u)=\exp \left(-\alpha_{l} v\|\mathbf{h}\|^{2}\right) \exp \left(-\beta_{l} v|u|^{2}\right)$ is a covariance function for any positive $v$. Thus, it suffices to show for which values of $\left(k_{1}, \ldots, k_{n}\right)$ the sum $\sum_{l=1}^{n} k_{l} \mathrm{C}_{s, t}^{v, l}$, belonging to the GSP class, is positive definite. From Proposition 1 and Example 1 it can be easily verified that, under condition (8), $\sum_{l=1}^{n} k_{l} \mathrm{C}_{s, t}^{v, l}$ is positive definite and so is the positive mixture represented by the integral. Thus the last summand in equation (10) is negative definite. 
For the part 2), it is sufficient to follow the same steps of equation (10) with variogram (7), and arguments of the previous paragraph with $\mathrm{C}_{s, t}^{v, l}(\mathbf{h}, u)=\exp \left(-\alpha_{l} v\|\mathbf{h}\|\right)$ $\exp \left(-\beta_{l} v|u|\right)$, and taking into account that the exponential covariance is a particular case of the Matérn family. Thus, the proof is completed.

Example 2. Let us propose as a spatial or temporal variogram a novel structure, which has never been considered, to our knowledge, in geostatistical literature. Berg and Pedersen (2001) showed that the function

$$
f(x)=\frac{\log \Gamma(x+1)}{x \log x}
$$

is a Bernstein function. This function has attracted the attention of various mathematicians in the past, among them Anderson and Qiu (1997). Using the scale invariance property with respect to a positive parameter $\alpha$, it is easy to see that

$$
\psi\left(\|\mathbf{h}\|^{2}\right)=\frac{\log \Gamma\left(\alpha\|\mathbf{h}\|^{2}+1\right)}{\alpha\|\mathbf{h}\|^{2} \log \left(\alpha\|\mathbf{h}\|^{2}\right)}
$$

is a valid variogram in any d-dimensional Euclidean space $\mathbb{R}^{d}$ for any positive $\alpha$. This variogram has the interesting property that it depends on one parameter only, so that estimation procedures for the variogram can be easier. Unfortunately, this variogram does not allow for the control of the level of smoothness of the process. This problem can be overcome by considering the logarithmically completely monotone function

$$
g(x)=\frac{(\Gamma(\alpha x+1))^{1 / \alpha x}}{\alpha x}\left(1+\frac{1}{\alpha x}\right)^{x}
$$

Berg (2004) showed that a function $g$ is logarithmically completely monotone if and only if $g^{\delta}$ is a completely monotone function for all positive $\delta$. Thus the function

$$
\varphi\left(\|\mathbf{h}\|^{2}\right)=\frac{\left(\Gamma\left(\alpha\|\mathbf{h}\|^{2}+1\right)\right)^{\delta / \alpha\|\mathbf{h}\|^{2}}}{\left(\alpha\|\mathbf{h}\|^{2}\right)^{\delta}}\left(1+\frac{1}{\alpha\|\mathbf{h}\|^{2}}\right)^{\delta\|\mathbf{h}\|^{2}}
$$

is a permissible covariance function for any d-dimensional Euclidean space $\mathbb{R}^{d}$, having the desirable property that it allows to control the level of smoothness of the process away from the origin.

Coming back to variograms, Berg (2004) showed that (11) is a Stieltjes transform, which is a stronger statement than that of complete monotonicity. This fact can be used jointly with Berg and Pedersen (2001, Prop. 1.3) by which if $\varphi$ is a Stieltjes transform, then $\psi=1 / \varphi$ is a Bernstein function. Finally, considering that if $\varphi$ is a Stieltjes transform, then $1 / \varphi(1 / x)$ and $(1 / x \varphi(x))$ are still Stieltjes transforms, we obtain that various novel forms of variograms can be proposed. For instance, the function

$$
\psi\left(\|\mathbf{h}\|^{2}\right)=\frac{\left(\alpha\|\mathbf{h}\|^{2}\right)^{\delta}}{\left(\Gamma\left(\alpha\|\mathbf{h}\|^{2}+1\right)\right)^{\delta / \alpha\|\mathbf{h}\|^{2}}}\left(\frac{\alpha\|\mathbf{h}\|^{2}+1}{\alpha\|\mathbf{h}\|^{2}}\right)^{\delta\|\mathbf{h}\|^{2}}
$$


is a permissible variogram on any d-dimensional Euclidean space $\mathbb{R}^{d}$. All the proposed variogram structures can be used in order to apply Proposition 2. Thus, setting $\alpha=1$, without loss of generality, the following structures

$$
\begin{aligned}
\gamma_{s, t}(\mathbf{h}, u)=\sum_{i=1}^{n} & k_{i} \frac{\left(\alpha_{i}\|\mathbf{h}\|^{j}+\beta_{i}|u|^{j}\right)^{\delta}}{\left(\Gamma\left(\alpha_{i}\|\mathbf{h}\|^{j}+\beta_{i}|u|^{j}+1\right)^{\delta /\left(\alpha_{i}\|\mathbf{h}\|^{j}+\beta_{i}|u|^{j}\right)}\right.} \\
& \times\left(\frac{\alpha_{i}\|\mathbf{h}\|^{j}+\beta_{i}|u|^{j}+1}{\alpha_{i}\|\mathbf{h}\|^{j}+\beta_{i}|u|^{j}}\right)^{\delta\left(\alpha_{i}\|\mathbf{h}\|^{j}+\beta_{i}|u|^{j}\right)}
\end{aligned}
$$

are valid intrinsically stationary spatio-temporal variograms defined on $\mathbb{R}^{d} \times \mathbb{R}$ under the prescribed constraints, respectively (8) and (9), for $j=1,2$.

\section{Conclusions and discussion}

The results presented in this paper can be useful for those disciplines, such as biology, hydrogeology and engineering, which are interested in the use of negative covariances for space time data. This research could be readdressed in the future to the solution of other problems. For instance, we considered as primary necessities the points 1 and 2 presented in the introduction, but other research aspects could be considered. For instance, a critical point to be taken into account is the consideration of zonally anisotropic behaviours in the spatial component of the space time covariance.

Instead of using Bernstein's formula (5), one can use the Lévy-Khinchine formula for conditionally positive definite functions (see e.g. Sasvári, 1994) to get linear combinations of variograms (with possibly negative weights) which are variograms as well. By choosing special variograms a table like Table 1 could be computed, using similar arguments as in Proposition 1.

\section{References}

[1] Anderson, G. D. And Qiu, S.-L. (1997). A monotonicity property of the gamma function. Proceedings of the American Mathematical Society, 125, 3355-3362.

[2] Berg, C. And Forst, G. (1975). Potential theory on locally compact abelian groups. Springer, New York.

[3] Berg, C. And Pedersen, H. L. (2001). A completely monotone function related to the Gamma function. Journal of Computational and Applied Mathematics, 133, 219-230.

[4] Berg, C. (2004). Integral representation of some functions related to the Gamma function. Mediterranean Journal of Mathematics, 1, 433-439.

[5] Bochner, S. (1933). Monotone funktionen, Stieltjes integrale und harmonische analyse. Mathematische Annalen, 108, 378-410. 
[6] Chilès, J. P. And Delfiner, P. (1999). Geostatistics: Modelling Spatial Uncertainty. Wiley, New York.

[7] Christakos, G. (2000). Modern Spatiotemporal Geostatistics. Oxford University Press, Oxford.

[8] Cressie, N. A. C. And Huang, C. (1999). Classes of nonseparable, spatiotemporal stationary covariance functions. Journal of the American Statistical Association, 94, 1330-1340.

[9] De Cesare, L., Myers, D. E. and Posa, D. (2000). Product-sum covariance for space-time modeling: an environmental application. Environmetrics, 12, 11-23.

[10] De Cesare, L., Myers, D. E. And Posa, D. (2001). Estimating and modeling space-time correlation structures. Statistics and Probability Letters, 51, 9-14.

[11] De Iaco, S., Myers, D. E. And Posa, D. (2001). Space-time analysis using a general productsum model. Statistics and Probability Letters, 52, 21-28.

[12] Dimitrakopoulos, R. and Luo, X. (1994). Spatiotemporal Modeling: Covariances and Ordinary Kriging System, Geostatistics for the Next Century, R. Dimitrakopoulos (Ed.), Kluwer Academic Publisher, 88-93.

[13] Gneiting, T. (2002). Nonseparable, stationary covariance functions for space-time data. Journal of the American Statistical Association, 97, 590-600.

[14] Janauer, G. A. (2001). Is what has been measured of any direct relevance to the success of the macrophyte in its particular environment? O. Ravera (Ed.) Scientific and legal aspects of biological monitoring in freshwater, Journal of Limnology, 60 (Suppl. 1), 33-38.

[15] Ibáñez-Gual, M. V. And Simó, A. (2004). Conditional and unconditional simulation of healthy patients' visual fields. Biometrical Journal, 46, 404-419.

[16] Levinson, S. J., Beall, J. M., Powers, E. J. and Bengtson, R. D. (1984). Space-time statistics of the turbulence in a Tokamak edge plasma. Nuclear Fusion, 24, 527-540.

[17] MA, C. (2002). Spatio-temporal covariance functions generated by mixtures. Mathematical Geology, 34, 965-974.

[18] MA, C. (2005a). Linear combinations of space-time covariance functions and variograms. IEEE Transactions on Signal Processing 53, 857-864.

[19] MA, C. (2005b). Spatio-temporal variograms and covariance models. Advances in Applied Probability, 37, 706-725.

[20] Matérn, B. (1986). Spatial Variation. 2nd edition, Springer, Berlin.

[21] Mitchell, M., Genton, M. G. and Gumpertz, M. (2005). Testing for separability of space-time covariances. Environmetrics, 16, 819-831.

[22] Myers, D. E. And Journel, A. (1990). Variograms with zonal anisotropies and non-invertible kriging systems. Mathematical Geology, 22, 779-785.

[23] Pomeroy, J. W., Toth, B., Granger, R. J., Hedstrom, N. R. and Essery, R. L. H. (2003). Variation in surface energetics during snowmelt in a subarctic mountain catchment. Journal of Hydrometeorology, 4, 702-719. 
[24] Porcu, E., Saura, F. and Mateu, J. (2006). New classes of covariance and spectral density functions for spatio-temporal modelling. Stochastic Environmental Research and Risk Assessment. To appear.

[25] Rohuani, S. And Hall, T. J. (1989). Space-time Kriging of Groundwater Data. Geostatistics, Armstrong, M. (Ed.), Kluwer Academic Publishers, 2, 639-651.

[26] Sasvári, Z. (1994). Positive Definite and Definitizable Functions. Akademie Verlag, Berlin.

[27] Schoenberg, I. J. (1938). Metric spaces and completely monotone functions. Annals of Mathematics, 39, 811-841.

[28] Shkarofsky, I. P. (1968). Generalized turbulence space-correlation and wave-number spectrumfunction pairs. Canadian Journal of Physics, 46, 2133-2140.

[30] Vecchia, A.V. (1988). Estimation and model identification for continuous spatial processes. Journal of the Royal Statistical Society B, 20, 370-373.

[30] Xu, Z.-W., Wu, J., Huo, W.-P. And Wu, Z.-S. (2003). Temporal skewness of electromagnetic pulsed waves propagating through random media with embedded irregularity slab. Chinese Physics Letters, 20, 370-373.

[31] Xu, Z.-W., Wu, J. AND Wu, Z.-S. (2003). Statistical temporal behaviour of pulse wave propagation through continuous random media. Waves Random Media, 13, 59-73.

[32] Yaglom, A. M. (1987). Correlation theory of stationary and related random functions. Springer, New York.

[33] Yakhot, V., Orszag, S. A. and She, Z.-S. (1989). Space-time correlations in turbulence Kinematical versus dynamical effects. Physics of Fluids, 1, 184-186. 